\title{
myTIS: a MAS-based TIS for public transport users
}

\author{
N. M. Gomes Rocha ${ }^{1,3}$, D. F. L. $\operatorname{Santos}^{2}$ \& R. J. F. Rossetti ${ }^{2}$ \\ ${ }^{1}$ Department of Civil Engineering, Faculty of Engineering, \\ University of Porto, Portugal \\ ${ }^{2}$ Department of Informatics Engineering, \\ Artificial Intelligence and Computer Science Laboratory (LIACC), \\ Faculty of Engineering, University of Porto, Portugal \\ ${ }^{3}$ PROEC - Projectos, Estudos e Construções Lda., Braga, Portugal
}

\begin{abstract}
This paper reports on the development of myTIS, which is a multi-agent based architecture to support public transport users in planning and executing their trips on a daily basis. Commuters that use public transport in their daily lives are frequently faced with problems, such as finding out what time the next bus service to a certain destination arrives or how to improve their travel experience to minimise the time spend in transit. On the other hand, non-commuters must address even more complicated issues, as they are not familiar with the network and would need continuous assistance throughout the trip realisation. These scenarios bring about interesting and challenging problems that suggest the adequacy of the multi-agent systems (MAS) metaphor. In a first abstraction exercise, it is very intuitive to identify such characteristics in the public transport domain. myTIS (where the TIS excerpt stands for traveller information system) is intended to be a cross-platform for public transport travellers, divided in terms of agents that cooperate to fulfil users' trip planning goals. Such a design decision is a requirement as the system must cover the whole decision-making process of a commuting plan. The methodological approach followed in this project includes a modelling phase, in which the requirements of the system and its main functionalities are defined. This phase is supported by an in-depth state-of-the-art review and the study of similar applications and results in the design of a new generation MAS-based traveller information system.
\end{abstract}

Keywords: route finding, multi-modal public transport, multi-agent systems, urban transport, sustainable transport, advanced traveller information systems. 


\section{Introduction}

In most dynamic systems, when something stops it means there is a problem with movement. In urban mobility this is traffic jams. It could have different causes, such as accidents or disrespect for traffic rules, but in the end they happen because too many cars are at a given point simultaneously, as argued by Nagatani [1]. So we have two variables, namely road capacity and the number of vehicles. Usually, urban planners increase road capacity in order to solve these problems. Actually, what happens is that the problem is transferred to another place. The highway/urban road connection is still a complex problem to tackle. Park and Ride, among other measures, is also a common answer in the attempt at reducing the number of vehicles inside urban areas. For commuters this usually works. However, if something goes wrong with the plan, bigger problems will probably be brought about. According to Pucher [2], non-commuters address even more complicated issues as they are unfamiliar with such measures. Other increased values of the private vehicle, such as availability, comfort and its use as a social status regulator, as suggested by Van Vaught et al. [3], make it even more difficult to give a reasonable answer to the problem of urban congestion. An equation to solve this problem must definitely add urban and public transport planning policies. In public transport planning there are two important issues to address, namely information and QoS (quality of service). In fact, today's information systems encompass a wide range of formats that ease communication and knowledge sharing, ranging from advanced traveller information systems or classical stop timetables to online journey planners, for instance. Despite the investments, public transport has not reached the door-todoor quality standard as yet. Considering this, we propose myTIS (my Traveller Information System) to provide users with a multi-modal public transport route advisor, with web interfaces for PCs and mobile interfaces for PDAs and similar portable devices.

The route finding problem is a very much developed area considering the number of papers being published within recent decades, although the multimodal route is much more complex than the single modal route. Therefore, we have designed the system to be applied to every public transport network and its interconnections. Dickson et al. [4] argue that this would require an object database model based on the MIRAS conceptual model for multi-modal transport networks modified to included regional or even international networks. We introduce GPS locations for origin input and mobility factors (such as shopping or going to cinema) destination input. We are also considering a solution for ticketing and route adaptation.

Such a complex scenario is inherently complex and due to its many autonomous and heterogeneous decision-makers (drivers, pedestrians and travellers in general) it has a nature of great uncertainty. Indeed, multi-agent systems are particularly well-suited to tackle problems in complex domains, whose entities are heterogeneous, exhibit social abilities and pursue either personal or collective goals, such as in public transport domains, as suggested by Schleiffer [5]. We intend to implement myTIS as a multi-agent traveller 
information system and expect this project will have a considerable social impact, especially due to its ability to contribute to the improvement of the traffic condition in major urban areas in opposition to the ever increasing use of private cars. Indeed, public transport has been increasingly misused as private vehicles have gained the preference of most users. Despite their higher cost, private vehicles offer other facilities such as availability, door-to-door services and comfort. myTIS is expected to draw the attention of commuters and noncommuters back to public transport as many of its disadvantages can be minimised, if not suppressed, as users will have access to more accurate and upto-date information about their trip options whenever a decision for public transport is to be made.

This paper is organized as follows. Section 2 presents the modelling phase where background and related work are presented, such as the main functionalities. Section 3 introduces architectural design in which technological issues are addressed and the implementation model is presented. Section 4 discusses opportunities for further research and market opportunities. We will conclude our paper in Section 5.

\section{Modelling phase}

\subsection{Background and related work}

The intention of this project is to develop a cross-platform for public transport travellers, allowing pre-trip and en-route re-planning, covering the whole decision-making process of a commuting plan. It is also applicable to noncommuters, such as tourists or occasional travellers, by continuously assisting them throughout the trip realisation. It has to be available in different media support formats. At the end of the day, a traveller should be able to plan and pay for his entire trip using only public transport modes, from Red Square, Moscow to Fifth Avenue, New York.

At an urban level the passenger should be able receive the most adequate route considering cost, time and other aspects updated with traffic information. At public transport company level, transport planners will receive a precise and detailed origin-destination matrix.

Public transport routing has been widely studied; however, en-route replanning, through mobile devices, featuring data updating and a ticketing system, is not known to be available as yet to the best of our knowledge. There are some popular route advisory web portals, such as Transport for London Journey Planner, Oporto journey planner Itinerium, Hong Kong MIRAS (Multi-modal Intelligent Route Advisory System), and even Google Transit, which support worldwide public transport networks. Moreover, the internal mechanisms of all these systems are trade secrets and we could only try to realise them from the user's perspective. Still, these systems require much input from the user so as to provide them with accurate results. Also, some of them are restricted to one mode of transport, or only one urban area. There are also some questions about the relation between the quality of inputs and the quality of the output as in some 
systems you could arrive $3 \mathrm{~km}$ away from your final destination if it is located in a $3 \mathrm{~km}$ length road.

The most important issue of any application involving networks is choosing the accurate shortest path algorithm, according to Zhan [6]. Recent studies suggest three as the most popular algorithms, which are the graph growth algorithm implemented with two queues, the Dijkstra algorithm implemented with approximate buckets, and finally the Dijkstra algorithm implemented with double buckets.

The first algorithm mentioned above is considered to be the fastest for one-toall shortest path problem. Nonetheless, Dijkstra can be terminated as soon as the shortest path distance is obtained if the goal is a one-to-one or one-to-some path, as explained in Zhan et al. [7].

\subsection{Functionalities}

In a web server or in a mobile device, passengers should be able to plan trips before and during their journeys in multi-modal public transport. We expect that web services could be useful for pre-trip planning. En-route re-planning is expected to be made on mobile devices. On both environments, the input of the origin and destination could be made by texting, for example, a name of a street, station, place of interest and mobility factors, or by clicking in a map the desired position. In the mobility factors area, we expect it works as a wishes box where passengers could input an action rather than a place. On mobile devices the origin input could automatically be generated by GPS geo-localization. Planned trip output could be graphic or sonorous. Basic timetable consultation should also be available on both platforms.

Passengers could also create a profile where basic information and a personal calendar, where all daily tasks, expected duration and localization, could be saved. The application should be able to develop a trip plan considering this information, and readjust it if something changes. It should also be possible to generate a file with a trip planning to send to other agents in different formats, as well as could a search historic be stored for further reference and improved decision-making.

Mobile platform planning on should have route and GPS tracking system and also intelligent abilities. Route updating means that during the planning phase it should be considered the passenger position and characteristics, for example distance to bus stop or passenger age (as it may most certainly affect walking speed), and the transport mode status, for example if it is delayed or anticipated. This requires the optimization of existing advanced traveller information system, meaning that walking phase has to be included in the trip planning. Featuring the system with GPS tracking, if the passenger misses any mode change in the route, the application should recalculate it using only public transport means. Intelligent abilities should include learning capabilities that will be important in an initial implementation phase. It means that if the system does not recognise a route, it would ask users what the mode of transport is, origin and destination and basic timetable to infer possible alternatives for future use. 
There is also the possibility to buy the tickets using an optimized version of the existing mobile banking services. With that in mind, it is possible to devise and implement features for the multi-modal trip to be used as a ticketing service.

The system has also back office services, in which it is possible to develop an origin/destination matrix using data gathered from multiple users searching the system in a specific region of the urban network. Data mining and data fusion tools are also being studied that will use such information to predict and infer opportunities, overcrowding situations, misusing or other unexpected effects in public transport networks. Other service tools, such as login or users' management, and network tools, such as add/change/remove a bus line, must also be available from a system management perspective.

\section{Architectural design}

\subsection{Technological issues}

Without mortgaging future developments on technological demands of our system, we can forward that for the current stage we are using Windows Mobile ${ }^{\mathrm{TM}}$ Compact dot-NET Framework 3.5. Our option for such a software framework is mainly due to its interoperability, allowing access to many functionalities implemented in other environments. Another important aspect of this framework is the Common Language Runtime, which is able to reduce our constraints about specific CPU capabilities under which the application will run.

Among other programming languages, we have opted to implement the current prototype in $\mathrm{CH}$, as it is completely compatible with the dot-Net Framework. As for the database management system, we are currently using PostgreSQL as an object-relational system with powerful add-ons, not only for geographical objects manipulation, but also for shortest-path algorithms implementation through PostGIS.

We are also implementing an XML-based description document as an extensible language in order to serialize data among different modules of the application. However, some technological limitations are being faced using Windows Mobile ${ }^{\mathrm{TM}}$ to run JavaScript ${ }^{\mathrm{TM}}$ maps by using shape files of the streets.

\subsection{Implementation model}

We are testing different implementation models, but our framework architecture is basically as depicted in figure 1 , as follows.

Future developments will definitely bring more complexity to this model. Nonetheless, we expect to maintain simplicity and objectivity for public transport users, which will not need to deal directly with technological issues.

We also expect to totally cut the costs for the final user, or at least transfer it to other stakeholders. However it is still a far goal to pursue, as we have been considering it at this design phase. 


\section{Future research and market opportunities}

\subsection{Future research}

At the current stage, we are working on the proof of the concept. We have to keep on implementing as much functionalities has we are able to. After that we expect to find co-partners to implement multi-background functionalities. For example, the ticketing and paying system is right now our Pandora box in terms of operation and security. Further we intend to bring the system to the market, considering possible merging with settled companies.

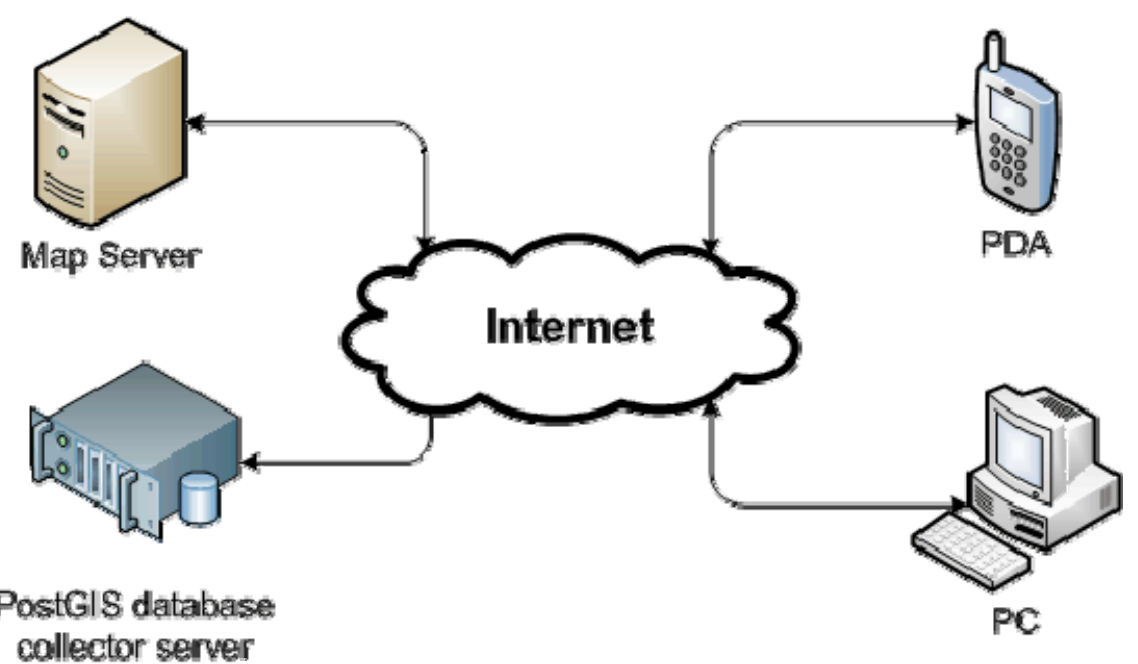

Figure 1: Basic implementation model.

\subsection{Market opportunities}

In a university environment survey, with different transport modes users, including private vehicle, we receive an $85 \%$ of positive answers to the question: "Would you use a service that allows you to plan public transport trips, available from your phone?" We also found that $41 \%$ of private vehicle users would consider public transport if they had access to a system with those characteristics. We know that the dimension and environment could significantly affect the results although it gives some indications such a system will play a major role in commuters and other public transport users' lives.

Mobile phone or Internet operators, GPS or software producers and public transport operators might improve their services with an application like myTIS by applying to their clients' accurate data about public transport, improving the quality of the users' mobility choice. 


\section{Conclusion}

This paper presented myTIS and its current development stage. Considering our best knowledge of the main differences between existing systems and our approach, GPS location for the origin input, "wishes" input for destination, personalised environment, system learning abilities, ticketing service and origin/destination matrix development are key features that will put public transport options back into commuters and non-commuters consideration and will foster the development of greener, smarter and more sustainable urban environments.

The current stage of development of myTIS framework includes the study of similar applications and the implementation of basic functionalities in order to achieve a concept proof.

Next steps in the conceptualisation of our system will include the implementation of fully agent-based modules that will interact with the end-users to enhance their experience in using public transport, emphasising their perception of costs on an individual basis. This is a must feature to be accomplished in such systems and it is definitely a key ingredient to captivate users for a multi-modal transport experience.

\section{References}

[1] Nagatani, T., The physics of traffic jams. Rep. Prog. Phys. 65, pp. $1331-$ 1386, Institute of Physics Publishing, 2002.

[2] Pucher, J., Urban Travel Behaviour as the outcome of public policy: The example of modal-split in western Europe and North America, APA Journal, pp. $509-520,1988$.

[3] Van Vugt, M., Van Lange, P.A.M., Meertens, R.M., Commuting by car or public transportation? A social dilemma analysis of travel mode judgements, European Journal of Social Psychology, Vol. 26, pp. 373 - 3951996.

[4] Dickson, K.W.C., Oliver, K.F. L., Ho-fung, L., Au, E. W. K., May, C. W. W., A multi-modal agent based mobile route advisory system for public transport network, Proceedings of the 38th Hawaii International Conference on System Sciences, pp. 1 -10, 2005.

[5] Schleiffer, R., Intelligent agents in traffic and transportation. Transp. Research C10(5-6):325-329. 2002.

[6] Zhan, F. B., Three fastest shortest path algorithms on real road networks: Data Structure and Procedures, Journal of Geographic Information and Decision Analysis, vol. 1, no. 1, pp. 69-82, 2001.

[7] Zhan, F. B., Noon, C. E., Shortest path algorithms: an evaluation using real road networks, Transportation Science, vol. 32, no. 31, 1998. 